\title{
Palaeontologie und
}

\section{Descendenzlehre.}

\section{ש Vortrag}

gehalten in der

allgemeinen Sitzung der naturwissenschaftlichen Hauptgruppe der Versammlung deutscher Naturforscher und Aerzte in Hamburg am 26. September 1901

VOI1

\section{Ernst Koken,}

Professor der Geologie und Palaeontologie in Tübingen.

— Mit 6 Figuren im Text.

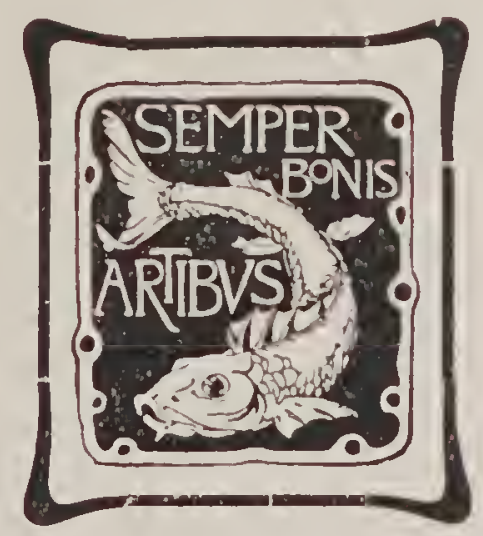

Verlag von Gustav Fischer in Jena. 
Alle Rechte vorbehalten. 
Durch die ersten Erfolge der im Anfange des 19. Jahrhunderts aufblühenden historischen Geologie, durch die Beobachtungen über den Zusammenhang der grossen, versteinerungsführenden Formationen und über das Ausharren mancher Arten durch mehrere geologische Zeitabschnitte wurde das alte biblische Dogma schwer erschüttert. Der Ausbau der Schöpfungslehre mit Berücksichtigung jener Thatsachen führte zu immer komplizierteren Annahmen. Die Kataklysmenund Katastrophentheorie, welche man noch mit den Schöpfungstagen der Mosaischen Lehre in Einklang zu bringen versucht hatte, erwies sich in ihrer starren Form als unhaltbar und sie verlangte eine solche Menge von Zusatzparagraphen, dass die Ueberzeugung, es müsse cine einfachere Auslegung des Werdeganges des Lebens geben, sich immer fester einnistete.

Lamarck's genialer Versuch, dem Gedanken einer von den einfachsten Gesetzen geleiteten Entwickelung Eingang zu verschaffen, wurde von der Majorität erstickt, aber in allen Ländern, wo Palacontologie getrieben wurde, glimmten die Funken seiner Lehre nach.

Wie ein Sturmwind ging dagegen die von Darwin entfachte Bewegung durch unsere Wissenschaft. I.üngst zusammengetragene Beobachtungen über die Tiergeschlechter früherer Erdperioden erschienen in neuem Lichte, und mit erhöhtem Interesse wurden ihre Beziehungen zu einander crörtert.

Dass viele der damals entworfenen Stammbäume wesenlos und viele Folgerungen unhaltbar waren, hat sich bald gezcigt, aber dennoch ist die Ueberzeugung von der inneren Berechtigung der Abstammungslehre in der Palaeontologic stärker gefestigt denn je, und wenn die Selektionslehre, wie sie Darwin geschaffen hat, unter 
den Palaeontologen weniger Vertreter hat und die Form der Abstammungslehre, wie sie allmählich sich bei uns entwickelt hat, mehr auf L,amarck zurückweist, so ist doch der Name Darwin's inniger und unvergesslich mit unserer Wissenschaft verbunden.

Der- Palaeontologe ist nicht in der Lage, über Vercrbung und Variabilität Versuche anzustellen — sein Material ist ein totes. Er kann auch seine Untersuchungen nicht auf beliebige Teile der früheren Organismen ausdehnen, denn meist liegen ihm nur die den Körper stützenden oder schützenden Hartgebilde und auch von diesen oft nur Fragmente vor, die mühsam in Zusammenhang gebracht werden müssen. In vielen Gesteinen, und leider gerade in den ältesten, wo wir mit erhöhtem Interesse den Formen des Lebens nachspüren, sind durch geologische Vorgänge, Faltungen und Schieferung, Streckung und Pressung des Gesteins, durch allmählich aufsteigende Erhitzung und chemische Umwandlung oder durch Kontakt mit glühenden Magma- und Lavamassen die Versteinerungen entstellt oder ganz zerstört.

Dennoch, und trotzdem noch viele andere Umstände eine grosse Lückenhaftigkeit des Gesamtmaterials bedingen -- es sei nur auf die Schwierigkeit der Erhaltung von Tiefseetieren und auf die nur unter gewissen Umständen mögliche Erhaltung der auf dem Lande sterbenden Organismen verwiesen — dennoch ist das Material ein ausserordentlich grosses und dadurch, dass es zeitlich genau geordnet ist, ein unschätzbar wertvolles. Zum exakten Beweis der Abstammungslehre wollen wir es gar nicht verwenden. Wir stellen uns auf den Boden der Descendenzlehre, die den komplizicrten Erscheinungen in der Aufeinanderfolge der Organismen gerecht wird und unser Kausalbedürfnis befriedigt.

Wenn wir als Palaeontologen zum Ausbau der Descendenzlehre beitragen wollen, so müssen wir den Vorteil ausnutzen, dass wir die Organismen in ihren Veränderungen durch eine ungcheucre Zeit begleiten können und dass wir zugleich in der Lage sind, das Bild der Erdgeschichte, der Entwickelung der physikalischen Bedingungen, welche die Organismen umgaben, anregten und beherrschten, zur Seite zu stellen. 
Der Zug des Todes ordnet sich in einer Weise, welche z.um Nachdenken über diese Anordnung und über das einstige l,eben der Formen auffordern muss. I)ie Gesteine, in denen unsere Toten liegen, bewahren die Züge, welche damals die Frde kennzeichneten. Wir können die abradierten Falten der alten Gebirge und den steingewordenen Boden der Tiefsee betreten, und diese Gesteine halten der Betrachtung stand, wenn wir uns in das entlegenste Altertum verlieren, und begleiten uns an die Schwelle der Gegenwart.

Unsere weitere Aufgabe ist, zu ergründen, wie in dieser Folge der Organismen die Fäden des Zusammenhanges geschürıt sind.

Wir untersuchen den (iang der Entwickelung in grösseren Abteilungen, wir verghtophe die verschiedenen Stämme miteinander, wir fragen, ob Reihenfolge oder Richtung auf gewisse Impulse, sei es mechanische oder geologische, zurückgeführt werden kann.

Die Formen, unter denen die Entwickelung oder Descendenz uns entgegentritt, sind nicht das eigentliche Geset\%, welches den Zusammenhang bewirkt und regelt, aber für unsere Auffassung ist es von höchster Wichtigkeit, sie kennen und auseinanderhalten zu lemen.

Jedes Phylum zeichnet sich hierin vor dem andern aus und es ist nicht richtig, das im Einen als hervorstechend und massgebend erkannte auf die anderen zu übertragen.

Diese palaeontologische Methode hat uns mehr von Darwin entfernt, als in den ersten Jahrzehnten nach dem Erscheinen seines Werkes für möglich gehalten werden konnte. Damals wurde die Palaeontologie überrumpelt. Man griff kühn in ihre Vorräte hinein, um den lebenden Organismen die geforderten Ahnen zu geben, und noch viel kühner ergänzte man die Stammbäume nach den Vorgängen in der Ontogenie, nach dem bingenetischen Grundgesetze. Die Palacontologie, welche auf die Ergänzung ihres Materials durch glückliche Funde warten nuss, wurde eine Zeit lang mitgerissen.

Ganz allmählich entstand aber eine Strömung, welche die Palaeontologie von der eigentlich Darwin'schen Lehre entfernte. Der Kampf um's Dasein und die Naturzüchtung schienen an Bedeutung zurückzustchen hinter anderen Prinzipien, welche für Darwin nur 
auxiliäre sind, denen er, ohne sie ganz zu verwerfen, nur nebensächlichen Wert zuerkennt.

Es war wohl selbst manchen Palaeontologen, die sich für Darwinianer hielten, entgangen, dass die Pointierungen der allermeisten palaeontologischen Monographien nicht im eigentlich Darwin'schen Sinne ausfielen, dass die Art der Veränderungen meist auf Anpassung subjektives Anpassen), auf physikalische Beeinflussung und mechanische Wirkungen und Gegenwirkungen zurückgeführt wurde, dass mit dem Kampf um's Dasein, der so häufig genannt wurde, nicht Aer $z w i s c h e n$ den Individuen einer Art gemeint war, sondern jene allgemeine Konkurrenz zwischen ganz verschiedenen Arten, der viel mehr in Lamarck's Anschauungsweise eine Rolle spielt als in Darwin's Selektionsprinzip.

Man hat nicht auf Lamarck zurückgegriffen, weil man der Darwin'schen Lehre müde war, sondern weil die Betrachtung grosser Reihen von Fossilien (Säugetiere und Reptilien besonders) uns in jene Bahn der Erklärung verwies, die auch die seine war. Ohne das grosse thatsächliche Material vor Augen zu haben, hätte man schwerlich diese entschiedene Wendung gemacht.

Die Lamarck'schen Gedanken sind aber auch nicht bedingungslos übernommen, sondern sie sind verändert nach den eigensten Resultaten palaeontologischer Forschung. So entstand, und zwar zuerst und besonders in Nordamerika, wo die Zufuhr an neuem Material selbst die Arbeitskräfte der begeisterten Forscher überwältigt, die Neolamarck'sche Schule. Und auch hier erlebten wir, dass einer Lehre, welche rein mechanisch erklären wollte, metaphysische Zuthaten sich beimischten, wie überall, wo vollständige Abrundung der Weltanschauung das System krönen soll.

In den Lehren des Neolamarckianismus ist aber die Palaeontologie nicht stehen geblieben; sie sind nie zum Dogma geworden, wenigstens nicht bei uns, und es ist auch unnötig, ein Schlagwort zu erfinden, mit dem man die Entwickelungslehre, wie sie jetzt von vielen Palaeozoologen vertreten wird, bezeichnen soll.

Wir wollen nunmehr einiges aus dem überreichen Material herausgreifen. 
Von unserem Archiv ist freilich ein grosser Teil \%erstört und zwar besonders der die ältesten Urkunden umfassende. Dic Zeit geht auch an den festen Gesteinen nicht spurlos rorüber. Wir kennen im allgemeinen keine Versteincrungen, die älter sind als das cambrische Schichtensystem, aber wir kennen ungehenere Massen ron Gesteinen, die noch älter sind, die sich ursprünglich unter solchen Umständen angelegt haben, dass sie woht Fossilien einschliessen könnten, aber uns keine Sipur ron ihnen enthüllen.

Demnach kann es an sich nicht überraschen, wenn die cambrischen Fossilien nicht .primordiale" sind im eigensten Sinn des Wortes, sondern schon eine lange Entwickelung voraussetzen, und wenn bis auf die Wirbelticre die grossen Phyla des Tierreiches won den Pflanzen müssen wir zunüchst absehen) schon gesondert vertreten sind.

Möglich ist auch, dass die noch älteren ()rganismen erhaltungsfähiger Teile entbehrten.

Ueberblicken wir die (iesamtheit der fossil bekannten Tiere rom Cambrium bis zum Tertiär, in dessen letyter Phase wir gleichsam stehen, so ist der unmittelbare lindruck der les fiortschrittes und zwar eines Fortschrittes, der sich in den nach unserer Anschauung höheren Tieren um das Vielfarhe rascher geltend macht als in den niederen Gruppen. Der Fortschritt mag in vicler Beziehung nur ein Differenzierungsprozess sein, der die Einrichtungen der Organismen kompliziert und specialisiert, er ist aber auch nachweisbar an Sinnesorganen und speciell bei den Säugetieren an der Zunahme und Komplizierung des Gehirnes, wodurch eine direkte [Teberlegenheit in sinnlicher oder geistiger Beziehung hervorgerufen wird.

Am weitesten lässt sich die Geschichte des Wirbeltierstammes übersehen, denn diese spiclt fast ron ihren Anfängen an in den postcambrischen Zeiten; und wenn man von einem Zeitalter der Fische, Reptilien, Süugetiere spricht, so ist das nicht unberechtigt. Es ist ja möglich, dass die Anfänge der Reptilien viel weiter zurückreichen, als die jetzt bekannten Reste annehmen lassen, aber das wird nichts daran ändern, dass in Obersilur, Deron und Carbon die Fische die dominierende Abteilung die Vertebraten sind und dass die Säuge- 
tiere erst im Beginn der Tertiärzeit die Reptilien in der Herrschaft ablösen.

Auch in den anderen Stämmen sehen wir zu bestimmten Zeiten diese oder jene Gruppe auffallend breit entwickelt und im allgemeinen solche früher auftreten, bei denen wir die ursprüngliche Organisation voraussetzen können. Vor allem aber bemerken wir, dass die grösseren geologischen Formationen sich palaeontologisch scharf charakterisieren lassen, und doch sind sie durch keine Katastrophen geschieden und niemals reissen die Fäden des Zusammenhanges ganz ab; auch die grössten Abschnitte gehen hier oder dort kontinuierlich ineinander über, wie die palaeozoische Aera in der Salzkette Indiens unmerklich in die mesozoische hinübergleitet. Die Gattung Lingula lebte in den cambrischen und lebt noch in den gegenwärtigen Meeren, Atrypa reticularis geht wenig verändert vom obersten Untersilur bis in den Kohlenkalk, viele Foraminiferenarten haben ganze Serien geologischer Zeitabschnitte überdauert.

Wenn trotzdem die von uns stratigraphisch getrennt gehaltenen Zeiten auch in der Ausgestaltung des organischen Lebens deutlich getrennt bleiben, trotz des vorausgesetzten gleichmässigen Flusses der Entwickelung der Arten ihre Abstände halten, so führen wir dies gleichsam auf Interferenzen der biogenetischen und geologischen Entwickelung zurück, auf das Eingreifen von „Umgebungsreizen“, welche teils direkt, teils (besonders bei höheren Typen) durch Anstachelung der Instinkte wirken und in letzter Linie mit den grossen geologischen Veränderungen zusammenhängen.

Bei der Verwertung der Palaeontologie für die Descendenzlehre ging man nicht hiervon aus, sondern richtete das Augenmerk mehr auf die kontinuierlichen Reihen.

Es ist nicht schwer, die Beweiskraft einer solchen palaeontologischen Reihe anzufechten, indem man sich darauf bezieht, dass wir nicht die ganzen Tiere mit allen ihren Organen untersuchen konnten, sondern nur wenige Reste vorführen, dass einige wichtige Stadien des Entwickelungsweges nur aus dürftigen Resten erschlossen sind, dass wir in grösseren Reihen nicht von Art zu Art die Linien führen, sondern Gattung an Gattung reihen, also abstracta pro concretis, und 
schliesslich, dass die Palaeontologen selbst in der Ausrichtung ihrer Reihen dissenticren.

Wenn in dieser indirekten Weise diskutiert werden sollte, so muss man schliesslich die Frage aufwerfen, wie denn die Gegner der Descendenz, nachdem sie alle Beweiskraft der palaeontologischen Forschung abgetötet haben, sich die Reihenfolge der fossilen Arten und Gattungen ihrerseits zurechtlegen, welche Bedeutung sie dieser merkwürdigen Folge unterschicben, welche zugleich cine ganz beständige und gerichtete Aenderung der Organe verrät und so häufig zu einer physiologischen Vervollkommnung hinführt.

Ist auch nicht der ganze Körper erhaltungsfähig, so muss doch auch in den erhaltenen Skelettteilen der gesuchte Zusammenhang durch Abstammung zum Ausdruck kommen. Es liessen sich in der That zahlreiche Beispicle palacontologischer Reihen aufführen, deren einzelne Glieder schr eng verbunden sind und wo die Stärke oder Verschiedenheit der Grösse des Zeitabschnittes entspricht, der sie trennt.

Waagen hat sich eingehend, an den etwas weiter gefassten Begriff der Reihe bei Beyrich anknüpfend, mit solchen Zusammenhängen beschäftigt und für die zeitlich aufeinanderfolgenden Varietäten, durch welche allmählich der Typus einer Art umgestaltet wird, den Ausdruck Mutation eingeführt. Varietäten gruppieren sich um eine Art während ciner bestimmten Zeit, sind geologisch gleichzeitig - Mutationen durchragen die Zeit, ersetzen sich oder lösen sich $a b$ und stellen die Bindung zeitlich getrennter Arten, die kleinen Schritte, welche bei der Artbildung gemacht werden, dar. WVagen's Untersuchungen knüpften an Ammoniten an, doch lassen sich zahlreiche Beispiele auch aus anderen Klassen anführen. Nach Waagen bilden zwei durch solche Mutationen zusammengehaltene Formen nur eine gute Art; nach neueren Untersuchungen reicht der kontinuierliche Zusammenhang durch Mutationen auch oft darüber hinaus, aber nicht unbeschränkt.

Die Lückenhaftigkeit unserer palacontologischen Urkunden mag öfter eine Diskontinuität vortäuschen, wo in der Natur keine war, aber wenn wir die Summe unserer gegenwärtigen Erfahrung ziehen so scheint doch vieles darauf hinzuweisen, dass die gleichmässige 
Abschattierung einer kontinuierlichen Reihe nicht sehr weit reicht. Eine Verbindung grösserer Gruppen durch die kleinen Phasen der Mutationen gelang noch nie. Und selbst wenn wir von den Mutationen absehen und genetische Zusammenhänge konstruieren, wo die Arten unverbunden sind, aber doch nach dem Grade der Aehnlichkeit und der zeitlichen Folge aneinander gereiht werden können, dehnt sich ein solcher Zusammenhang fast nie über mehr aus, als dem Umfang einer Klasse des zoologischen Systems entspricht und lässt in jedem Falle die grossen „Phyla“ unberührt.

So kann man nach den vorliegenden Funden annehmen, dass mit Ausnahme der Monotremata alle Säugetiere von Formen ausgehen, welche den Insektivoren nahestanden, dass aus diesen sich primitive Carnivoren oder Creodontia ${ }^{1}$ ) entwickelten und erst von diesen aus die Spaltung in Marsupialia und Placentalia erfolgte. Auch die peripherisch stehende Gruppe der Edentaten kommt durch die Ganodontia zum Anschluss, die Phociden können wit Wahrscheinlichkeit von den Creodontia abgeleitet werden und auch für die Zahnwale erscheint eine derartige Abstammung möglich. In den letzteren Fällen muss man allerdings schon recht konstruktiv vorgehen, da das vorhandene Material äusserst lückenhaft ist, aber bei dem nachweisbar engen Zusammenhange anderer Säugetierlinien ist vorauszusetzen, dass diese Lücken nur zufällige sind und keinen Abbruch der Beziehungen bedeuten können.

Auch in anderen Klassen liess sich der phyletische Zusammenhang, den wir voraussetzen, bestätigen. so bei den eigentlichen Gastropoden; aber weder haben wir bisher fossile Formen nachweisen können, welche einen Zusammenhang der Scaphopoden und Pteropoden mit ihnen illustrierten, noch existieren auch nur andeutungs-

I) Die Creodontia sind nicht scharf von den Carnivoren geschieden und die Eigenschaften, die man als charakteristisch für sie aufführt, verlieren sich teils bei den jüngeren Formen, teils werden sie ro: den ältesten Carnivoren aufgenommen. Viele Merkmale können nur als transitorische beurteilt werden. Die fast glatten Hirnhemisphären der älteren Formen sind bei Hyaenodon im Oligocän schon intensiv gefurcht; Scaphoid, Lunare und Centrale verwachsen wie bei den Carnivoren. Und die ältesten echten Carnivoren haben relativ weniger gefurchte Hemisphären und "deutliche Nähte zwischen den genannten Élementen des Carpus. 
weise Uebergänge zu den Cephalopoden oder zwischen Gastropoden und Zweischalern. Da cliese Gruppen alle in das Cambrium zurückreichen, muss ihre Trennung rom gemeinsamen Stamm der Mollusken in noch früherer Zeit geschehen sein.

Nur sehr selten haben sich Uebergänge der Klassen und Ordnungen direkt nachweisen lassen: mit Recht betont claher Jaekel die Wichtigkeit einer Form wie Cystoblastus, welche die Abzweigung der Ordnung der Blastoidea aus den Cystideen nicht länger bezweifeln lässt.

Noch höhere Bedeutung hat Archaeopteryx für die Genealogie der Vögel. Sie giebt uns das Bild eines Vogels, wie man es zcichnen müsste, wenn man anerkennt, dass von den embryonalen und jugendlichen Merkmalen und Zuständen der lebenden Vügel ein 'Teil die ausgewachsenen Vögel der längst entschwundenen Jurazeit charakterisierte, und zeigt dazu eine Reihe reptilischer Nerkmale, wolche noch vorhanden sein müssen, wenn wir uns der Abzweigung der Vögel aus dem Reptilstamme nahe befinden.

Nach den Forschungen der letzten Jahrzehnte ist auch ein Zusammenhang der Reptilien mit den Säugeticren einerseits und durch die Stegocephalen mit den Amphibien andererseits annehmbar geworden. Oder wir können auch das Verhältnis sn deuten, dass wir den Stegocephalen eine centrale Stelle einräumen und ron ihnen über die Reptilien zu den Säugetieren die Verbindungen ziehen und dann wieder zu den Amphibien, deren Jugendzustand mit Kiemenatmung eine Neuerwerbung sein dürfte. Ganz abgesehen daron, dass wir hier schon vielfach subjektiv verfahren und grosse Lücken überspringen, ganz abgesehen daron, dass unter den Reptilien selbst die Zusammenhänge noch durchaus nicht klar gestellt sind und Chelonier, Ichthyosaurier, Pareiasaurier und Dinosaurier sehr scharf geschiedene Gruppen sind, deren Verbindungen crst gefunden sein wollen, sind wir damit vorläufig am Ende der Möglichkeit phylogenetischer Konstruktion angekommen.

Eine weite Kluft scheidet die Fische ron allen vierfüssigen Wirbeltieren, eine Kluft, die sicher durch die amphibische Lebensweise und Lungenatmung der Dipnoer auch nicht annähernd überbrückt wird. Die Dipnoer sind uralte Formen und haben manche wichtige 
Aehnlichkeit mit den holostomen Fischen ${ }^{1}$ ), ihre Differenzierung in lungenatmende Tiere hat einen terripetalen Charakter, während die Kiemenatmung der Amphibien eine sekundäre Erwerbung ursprünglich lungenatmender Tiere ist.

Irgend eine fossile Form, die als Uebergang vom Vertebratenstamm zu einem anderen Phylum gedeutet werden könnte, ist bisher

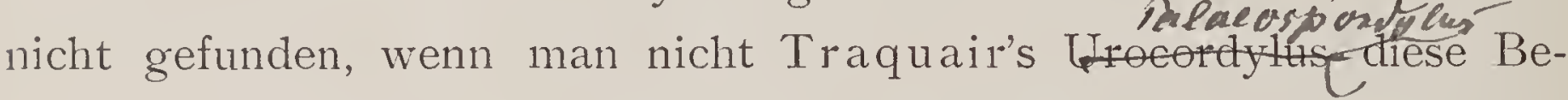
deutung beilegen will. Diese Reste sind aber oft so schattenhaft, dass eine präcise Beurteilung unmöglich ist.

Scharf getrennt reichen alle die grossen Phyla bis in das Cambrium zurück und aus jenen Zeiten, wo sie noch verbunden gewesen sein könnten oder wo sie sich aus gemeinsamer Wurzel abzweigten, fehlen die Berichte.

Wollen wir über die Art und Weise des Entwickelungsganges Studien machen, so müssen wir uns auf enger gefasste Gruppen beschränken, wo das Material eine Analyse der Stammesgeschichte erlaubt.

Die folgenden Bemerkungen stützen sich zunächst auf die Palaeontologie der Evertebraten, speciell der Mollusken.

Nach Darwin werden beliebige Varietäten erzeugt und die besten von der natürlichen Züchtung ausgewählt und zu neuen Arten ausgebildet. Da dies nur geschehen kann, wenn die Abänderung als überlegen sich erweist, so soll die Stammart untergehen, während die Extreme sich fortpflanzen. Die Palaeontologie kennt aber zahlreiche Fälle, wo die Stammart neben den Zweigarten bestehen bleibt, ja schliesslich noch persistiert, wenn diese wieder verschwunden sind - und das in einem eng umgrenzten Bezirk, wo die Principien der Isolierung und Wanderung nicht in Frage kommen.

I) Die Autostylie der Holocephalen und der Lungenfische wird von Dollo als eine Konvergenzerscheinung gedeutet, hervorgerufen durch ähnliche Entwickelung des Gebisses (Mylodontie. „Par conséquent - l'autostylie n'a rien de radicalement différent de l'hyostylie dont elle provient - et dont elle n'est qu'une adaptation à un régime triturateur très spécialisé - dans un but de consolidation de l'appareil masticatoire (Bull. soc. belge de Géol. IX, I 895, Mém. 79 ff., p. I Iо.) Das könnte sein, erklärt aber noch immer nicht die auffallende Aehnlichkeit in der Organisation des Gehörs, eines für die Aufdeckung verwandtschaftiicher Linien ausserordentlich wichtigen Organes, welches den Anpassungen wenig unterworfen ist. (Koken, Otolithenstudien. Zeitschr. d. deutsch. geol. Ges., I891, S. I56.) 
Hier knüpft die von mir als iterative Artbildung bezeichnete Erscheinung an ${ }^{1}$ ). Eine persistente Art treibt von Zeit und Zeit Varietäten, die gleichsam schwarmartig auftreten, während dazwischen mehr oder weniger lange Ruhephasen liegen. Ich beobachtete dies zuerst bei älteren Gastropoden, aber auch bei Craniaden, Pectiniden ${ }^{2}$ ) etc. sind Fälle iterativer Artbildung beschrieben.

Die Dauerformen können sich verbreiten und in verschiedenen Meeren ansiedeln und eine Periode der Artbildung durchmachen. Da die Varietätenbildung nicht unbeschränkt ist. so treten uns dann nicht nur in verschiedenen geologischen Zeiten, sondern auch in verschiedenen Provinzen Gruppen ähnlicher Arten entgegen. Die Worthenien nder L.ophospiren und die Raphistomen des baltischen und des nordamerikanischen Untersilurs, unter denen einige kaum zu unterscheiden sind, haben sich unter fast gleichen Bedingungen aus fast nder ganz gleichen Mittelformen entwickelt. Liei Annahme knmplizierter Wanderzüge eng verwandter Arten muss man sich daher grössere Reserve auferlegen, ats gewöhnlich geschieht.

Auch die sog. persistenten Typen, unter denen Lingula und Pleurotomaria so oft genannt werden, können in diesen Zusammenhang angeschlossen werden. Es sind durchaus nicht inclifferente Formen, die auf keinen Reiz reagieren; eine grosse Anzahl von Arten, die allerdings nur wenig differieren, setzt \%. B. die lingula-

1) Die Beobachtung, dass mitunter eine bestimmte Gestalt sich durch lange Perioden fast ungeändert fortsetzt, aber wederholt der Ausgangspunlit einer nach allen Seiten fortwuchernden Artbildung wird, wurde von mir i 889 in ,Entwickelung der Gastropoden vom Cambrium bis zur Trias" beschrieben. 1896 führte ich die Bezeichnung iterative Artbildung ein. „Diese Schwärne ron Varietiten und Arten liegen gleichsam stockwerkartig übereinander, ohne, wie es scheint, direkt genetisch rerbunden zu sein. Aehnliche Formen wiederholen sich, indem sie zu verschiedenen Zeiten aus dem konservativen Stammhalter hervorgehen, aber nicht, inden sie eine der anderen die Existenz gaben." (Jahrb. d. K. K. geol. Reichsanst. 1896, S. 40.)

2) „, Der Vola-Typus, mit vertiefter Unterschale und flacher Oberschale, tritt einmal im Lias, das zweite Mal in der Kreide und das dritte Mal in Tertiär auf. Zwischen Lias und Krreide und Kreide bis Oligocän klaffen riesige Lücken, aus denen uns von Vola keine Spur bekannt geworden ist. Die drei Vola-Typen sind trotz der Uebereinstimmung in einem Merkmal nicht miteinander direkt verwandt, sondern entstehen getrennt voneinander aus dem persistierenden Stamme der normalen Pectiniden." (Philippi, Sitz.-Ber. Naturt. Freunde, Berlin I 899, S. 89.) Vgl. auch Fir. v. Huene, Die silurischen Craniaden der Ostseelünder, S. 326 u. a. $1899^{\circ}$ 
Reihe zusammen. Bei Pleurotomaria ist die Amplitude des Variierens und der Artbildung schon grösser und man kann eine Anzahl Genera absondern - aber doch erhält sich der Typus mit grosser Zähigkeit und weicht in minimalen Abänderungen den umgestaltenden Faktoren aus. Auch hier erfolgt die Varietätenbildung in Intervallen und nicht ohne Beziehung zu geologischen Daten.

Der Kampf um's Dasein und die natürliche Auslese können in den bisher angeführten Fällen nicht stark eingegriffen haben, sondern die Artbildung scheint abhängig von der Konstitution und von aussen herantretenden Einflüssen.

Grösseres Interesse, als diese in kleinem Rahmen sich vollziehenden Vorgänge, rufen gewöhnlich die Fälle intensiver Abänderungen hervor, wie sie im Stamme der höheren Vertebraten beobachtet werden. Oft besprochen ist die merkwürdige Umformung des Reptiltypus bei den Ichthyosauriern und bei den Ornithosauriern, die Abstammung des Pferdes von fünfzehigen, höckerzähnigen Vorfahren u. a.

Im allgemeinen sucht man die Ursache so einschneidender Umänderungen in der Richtung des Nützlichen. Die Umänderung kann eingeleitet werden einmal durch ein aktives Sich-Anpassen, durch Wollen und Gewöhnen, was eine gewisse Herrschaft des Tieres über seinen Körper voraussetzt, dann aber auch durch eine passive Anpassung, wobei der Körper des Tieres abhängig ron der Umgebung (im weiteren Sinne) wird.

Das erstere ist möglich besonders dort, wo es sich um gant. allgemeine Funktionen, um die Art der Bewegung, wie Schwimmen, Fliegen, Laufen, handelt, und man kann die Geschichte der Huftiere als Beispiel heranziehen.

Der zweite Fall müsste zu schrankenloser Umbildung des Körpers führen, wenn nicht die dem Stamme eigene Konstitution die Führung übernimmt oder doch Grenzen zieht, wie es ja auch im ersten Fall geschieht. Als Resultierende aus Konstitution und Anpassung kommt eine Richtung der Entwickelung zu stande, welche eine Zeitlang konstant $z u$ sein pflegt. Hiernach werden in der Palaeontologie zuweilen die Genera gebildet, indem man alle 
Arten zusammenfasst, deren Charaktere auf eine bestimmte Richtung der Entwickelung zurückzuführen sind.

Man kann das nicht einfach mit Eimer "organisches Wachsen“ nennen, sondern es ist meist ein komplizierterer Vorgang und die Richtung ist weniger eine Tendenz- als cine Beharrungserscheinung.

Zweifellos vorhanden ist eine Prädisposition für gewisse Fälle des Variicrens und gerade das zeugt auch für den Einfluss der Konstitution. Von Arten, die sich in verschiedene Gegenden verbreitet haben, gehen fast dieselben Varietäten aus; wo der Schwäbische Jura am Hermon heraustritt, wiederholen sich im Ornatenthon auch die Spielarten des Ammonites hecticus. Schliesslich liegt ja auch dasselbe Moment der iterativen Artbildung zu (irunde.

Ist aber die Variabilität limiticrt, so hat auch die Abhängigkeit der Form von äusseren Einflüssen ihre Grenze und wird auch die Konvergenz beschränkt, welche genetisch sehr verschiedene Reihen infolge der Anpassung an ähnliche nder gleiche Bedingungen einander näher bringt. Von der Konvergenz werden besonders äussere Organe betroffen, während \%. B. solche, welche im Innern geborgen, den Beeinflussungen durch Bewegungsart und Medium entzogen sind, wie die merkwürdigen Otolithen der Knochenfische. weniger berührt werden.

Die Konvergenz zwischen einem Ichthyosaurus und einem Delphin bleibt cine ganz äusserliche, weil der Bauplan der Tiere zu weit rerschieden ist, dagegen führt die Konvergenz bei näher rerwandten Linien zu Erscheinungen, die schwieriger zu entwirren sind. So sind aus verschiedenen Linien der Selachier zu sehr verschiedenen Zeiten Rochenformen entstanden und wenn man die komplizierte Phylogenie dieser Gruppe nicht kennt, wird man hier eine systematische Einheit schaffen, die durchaus polyphyletisch genannt werden muss. Es lässt sich nicht verkennen, dass für viele Gruppen des bisher üblichen Systems der Auflösungsprozess, wenn anders wir ein „natürliches" System wollen, vorausgesagt werden kann, sowohl bei niederen Tieren (z. B. Pulmonata, Opisthobranchiata, Rhachiglossa, Taenioglossa, Ptenoglossa ctc.) wie bei höheren ( $\%$ B. Pharyngognathen, Physostomen). 
Dass aber selbst, wie die neuesten palaeontologischen Forschungen ergeben, eine scheinbar so geschlossene Gruppe wie die Feliden diphyletisch ist, muss allerdings auffallen. Eine Diphylie im engeren sinne d. h. eine Konvergenz heterogener I,inien, liegt allerdings nicht vor, sondern die zwei Stämme der Feliden kommen aus gemeinschaftlicher Wurzel, haben sich aber so wenig von einander entfernt, dass wir sie für einen halten konnten. Dennoch führen sie getrennt bis zum Eocän herab. Ein ganz unscheinbarer Spalt im System lässt sich eine enorme Zeit zuı ück verfolgen.

Die ausserordentlich geringe Distanzierung der beiden seit dem Eocän nebeneinander herlaufenden, in denselben Ländern heimischen Stämme der Feliden giebt aber vor allem eine Andeutung, wie wenig die natürliche Züchtung eine schon vorhandene Divergenz aliszugestalten vermag, wenn die Lebensweise die gleiche bleibt. Gerade bei Arten, welche nahe verwandt sind, gleiche Anforderungen an das Leben stellen und dieselben Gegenden bewohnen, sollte der Kampf ums Dasein nicht eine vollkommene Parallelentwickelung, sondern eine Differenzierung herauszüchten.

Diese ruhige, gleichmässige Fortentwickelung einer typischen Form wird noch bemerkenswerter, wenn wir mit ihr die Veränderungen kontrastieren lassen, welche den A enderungen der L ebensweise folgen. Hier sind mächtige Impulse, welche ganz Neues zu schaffen streben und um so mehr erreichen, je weiter die Instinkte und der Willen des Tieres ihnen entgegenkommen.

Die Ichthyosaurier sind unter den Reptilien die am meisten umgeänderten. Besonders im Hinblick auf das eigentümliche (Gliedmassenskelett hat man sich lange der Auffassung verschlossen, dass man es hier mit abgeleiteten Formen zu thun habe und vorgezogen, sie an den Anfang der morphologischen Reihe zu stellen. Baur erkannte in Mixosaurus eine Gattung; welche in der Bildung von Ulna und Radius noch an den Gliedmassenbau landbwohnender Wirbeltiere erinnert; Mixosaurus ist die alttriasische Form, Tchthyosaurus die jüngere - damit war die Richtung der Anpassung festgelegt. Man kann das auch aus der Analogie mit anderen Reptilien, welche infolge des Lebens im Meere Umgestaltung der Gliedmassen erlitten haben, folgern. 
Die Mosasaurier sind Abkömmlinge varanidenähnlicher Formen (Opctiosaurus), und selbst einige meerbewohnende Krokodilier der Jurazeit (Geosaurus, Rhacheosaurus) haben schanfelförmige Vorderextremitäten mit platten, breiten Knochen. Bei allen diesen tritt zugleich ein rertikales Schwanzflossensegel auf, ein kräftiger Propeller beim Durchschneiden der Wellen.

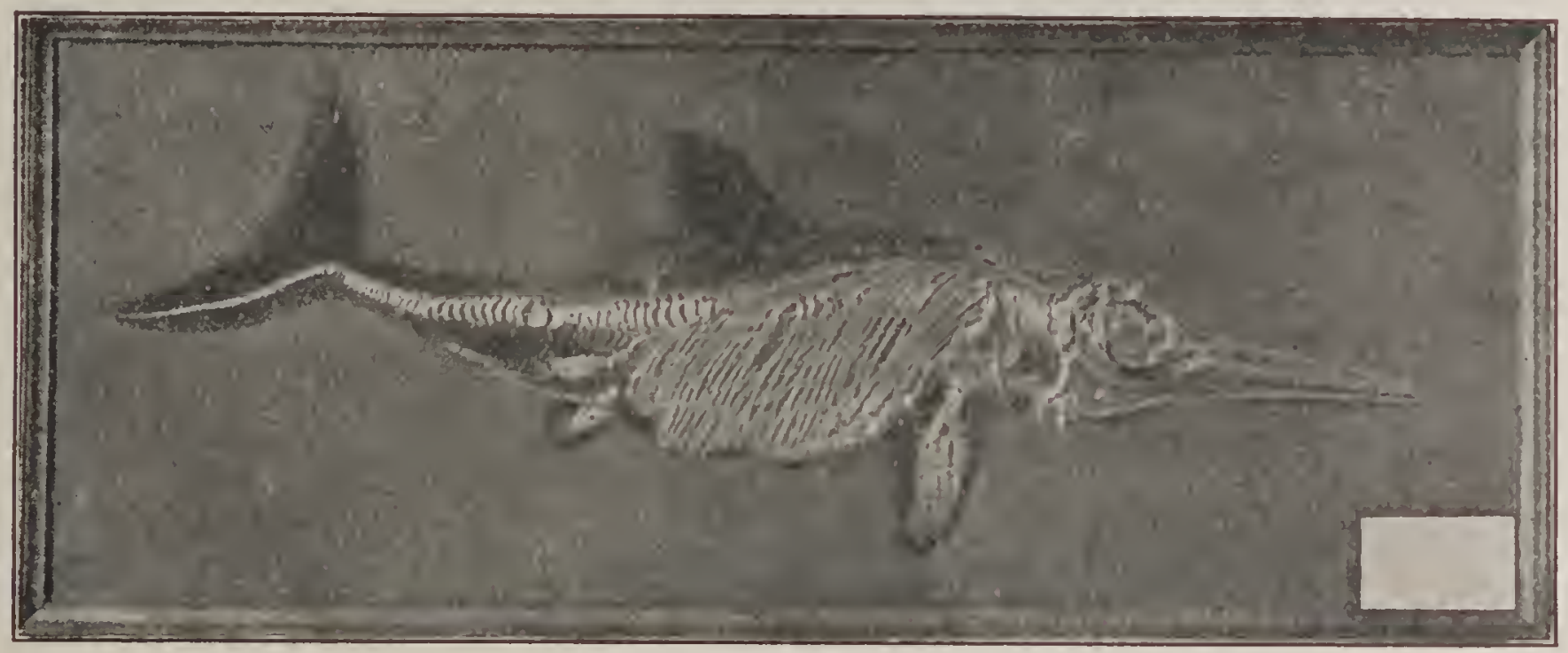

Fig. I. Ichthyosaurus triscissus Qu. Dis voilstindig erbaltene Fiemplar in der geologischen Sammlung der Universitül Tübingen (c. 1/15). Aus dem oberen Lias ( $)$ ) von Holzmaden in Württemberg. Man erkennt dentlich die grosse vertikal gestelle Schwan\%flosse, die Rückenflosse und einen bis zum Kopf sich erstreckenden Hatkamm, welcher durch kleine Knochenstüclic versteift wird. Die Extremitïten sind schaufelförmig, sog. ,Finnen“, und stecken in einer derben, häutigen Umhüllung.

Wir können diese und mit ihnen eng verknüpfte Einrichtungen bis zu einem gewissen (rrade als Iunktionen der L.ebensweise in einem gleichmässig wirkenden Medium, dem Meereswasser, auffassen. Man könnte also sagen, etwas Derartiges musste entstehen, wenn die Reptilien anfingen, sich schwimmend zu bewegen und ihre Beute $z u$ suchen.

Wenn wir aber unsere Blicke übcr die Gruppen der Ichthyosaurier, der Mosasaurier, der Thalattosuchia und der Plesiosaurier, welche ja auch zweifellos von landbewohnenden Reptilien abstammen, schweifen lassen, so müssen wir uns auch sagen, dass in der Ausführung der Anpassung sich Verschiedenheiten einstellen, welche nicht allein auf die Rechnung der verschiedenen Konstitution gesetzt werden können - denn in den ersten Anfängen müssen alle diese Linien ron Reptilien eine grosse Aehnlichkeit miteinander gehabt haben. 
Es sind auch wohl schwerlich so viel rerschiedcne und doch gleichwertige Nüancierungen des Nützlichsten nebeneinander denkbar, dass wir die verschiedene Organisation am Ichthyosaurus, Mosasaurus, Plesiosaurus auf das Wirken der Selektion zurückführen können. Viel näher liegt die Annahme, dass die Tiere von vornherein auf verschiedene Weise sich fortmubervegen suchten, dass also instinktives oder gerichtetes Wollen in erster Linie dafür verantwortlich ist, wenn die Anpassung bei Ichthyosaurus derart ausfiel, dass ein Hauptteil der Fortbewegung rom Schwanze übernommen wird, während bei Plesiosaurus mehr die Ruder herangezogen und entsprechend ausgestaltet sind.

Die Anpassung ist bei diesen Tieren so weit getrieben, dass eine Umkehr unmöglich, der Rückzug auf das Land abgeschnitten war. Das prägt sich auch aus in der Viviparität der Ichthyosaurier, einer für Reptilien nicht häufigen Erscheinung. Als die gewaltigen MosaSaurier und die mit furchtbarem Gebiss ausgestatteten Riesenhaie auftauchten (Ende der Kreidezeit), wurden die Ichthyosaurier ausgerottet. Die Anpassung wurde 7.um Verhängnis, weil sie in den Bau des Skelettes derart eingreift, dass die wichtigsten Teile gleichsam festgelegt wurden und einer Beanspruchung von anderer Seite nicht mehr nachkommen konnten. Diese Entwickelungsrichtung lag nicht im Bauplan des Tieres, sie ist ihm octroyiert. Anpassungen, bei denen die ganze Organisation gleichsam auf ein Ziel der Entwickelung festgelegt wird, können immer bei einer Verschiebung der Konstellationen zum Untergange der Gruppe führen; sie können also auch durchaus nicht einer weiterschauenden, teleologischen Ursache zugeschoben worden ${ }^{1}$ ).

I) Eine ebenso teleologische wie unlogische Auffassung liommt häufig genug zum Ausdruck. Wenn Dollo, indem er die Marsupialier von primitiven Placentaltieren ableitet, die Rückbildung der Placenta darauf zurückführt, dass das noch unvolikommene Organ beim Uebergang der Tiere zu der mit lebhaften Bewegungen verbundenen arboricalen Jebensweise Frühgeburten veranlasste, so mag das im Bereich des Denkbaren liegen. Falsch ausgedrückt aber ist der Satz: Et c'est, probablement, pour ériter ces accidents que cet organ embryonnaire a disparu. Wer hat hier den Zweck gesetzt? Das Organ - doch unmöglich. Das Tier, welches sich durch die rudimentäre Placenta belästigt und gefährdet fühlte? Ich halte es für möglich, dass ein Tier aus Instinkt oder mit Absicht seine Lebensweise ändert, aber ich halte es für mindestens schief ausgedrüclit, einem Tiere zuzuschreiben, 
Ganz auffallend ist in dieser Beziehung der Untergang der Ornithosaurier. Im Flugvermögen und in der Pneumacität der Knochen, deren Foramina beweisen, dass Ausstüipungen der Bronchien sich in sie hineinzogen wie bei lebenden Vögeln, waren sie schon in der Liaszeit auf einer Stufe angelangt, welche die Vögel erst viel später erreichten. Ihr Untergang mag damit zusammenhängen, dass keine Vorkehrung zum Wärmeschutz getroffen war, ein Mangel der Organisation, der besonders fühlbar wurde, als in Jura und Kreidezeit die Klimate der Erde sich schärfer accentuierten, der aber auch von vornherein den Flug in die höheren, kalten Regionen der I,uft ausschloss.

Eine der lehrreichsten Gruppen ist die der Huftiere, die heute in rerschiedene pflanzenfressende Gruppen geschieden sind, im älteren Eocän mit den Carnivoren noch zusammenhingen. Hier könnte man an ein rorschwebendes Ideal denken; konsequent nähern sich die Tiere einem Typus, der Schnelligkeit mit Festigkeit rereint und zugleich mit Sparsamkeit gebaut ist. Die Equiden sowohl wie die Paarhufer könnten nach dem Prinzip des selektiven Nutzens gezüchtet sein, aber Gewöhnung an einen bestimmten Gicbrauch der Gliedmassen und Zähne werden zu demselben Ziele führen und wir werden diese Erklärung bevorzugen, weil sie sich auch auf aberrante formen an-

dass es über den Schwund eines Organs beschliesst. Damit veriegt man die schaffende Kraft, die man durch die Descendenzlehre ausschliessen will, in deteriorierter Qualitït in die Machtsphäre des Individums.

Die Verstrickung in teleologische Anschauungen ist selbst bei einem so scharfen Denker und unübertroffenen Beobachter wie Kuwalewsky offenbar. Man vergleiche seine Ausführungen über die Reduktion der Zehen bei Anchitherium (Monographie der Gattung Anthracotherium, S. 161). Ich hele nur folgenden Passus herror: „Um diesen weiteren Schritt zu thun, d. h. um die Seitenzehen gänzlich abzuwerfen, muss man sie vorher unnüt\% machen, anders wird es keinen Grund zu ihrem Verschwinden geben; unnütz aber können die Seitenzehen nur in einem Falle werden, wenn sie den Boden nicht mehr berühren werden; folglich reduziert sich die nüichste Aufgabe darauf, die Seitenzehen vom Boden abzuheben." Und weiter wird dann ausgeführt, wie dies durch Verlängerung der ersten Phalange der Mittelzehe erreicht wird. Solitnge die Seitenzehen den Boden noch berïhren, sind sie nützlich, können also nicht abgestreift werden; sobald aber die Mittelzehe sich so verlängert, dass die Seitenzehen vom Boden abgehoben werden, wïrden sie nutzlos - und reduzierbar.

Hier ist in den „Organismus" eine ganze Welt weitausschauender Gedanken gelegt, und zwar ist die Rolle des zielstrebigen Ueberlegens nicht dem Individuum, sonderu der abstrakten, wesenlosen Art zuerteilt. 
wenden lässt, bei denen die Entwickelung in eine ganz neue Richtung einbiegt. Geringere Abweichungen stellen sich öfters ein.

Unter den bis in die Einzelheiten des Skeletts bekannten Rhinoceroten des nordamerikanischen Miocäns ist Teleoceras fossiger eine merkwürdige Form, im Habitus fast einem Nilpferd ähnlich. Der von kolossalen Rippen umgürtete, tonnenförmige Leib muss fast die Erde gestreift haben und steht im Missverhältnis zu den zwar stämmigen, aber sehr kurzen Beinen. An den Füssen verraten die fünf gespreizten Zehen und die Art des Muskelansatzes das Leben im Wasser. Der Umänderung der Lebensweise ist der Bau des Skelettes nachgefolgt.

Das Gegenstück liefert Hyracodon, den ungehörnten Aceratherien verwandt. Hier geht der Habitus der Rhinoceroten in den der Pferde über. Am Vorderfuss sind Humerus und Radius, die Carpalien und Metacarpalien, am Hinterfuss entsprechend Tibia, Metatarsalia und selbst die Phalangen verlängert oder höher. Der Rumpf mit kurzen, schmalen Rippen entspricht den schlanken Beinen, an denen nur die mittleren drei Zehen den Boden berührt haben. Hier haben wir ein offenbar schnellfüssiges Tier mit Eigentümlichkeiten, welche auch in der Osteologie des Pferdestammes sich zeigen - einen Bewohner der Prärien oder offenen Landschaft. In allen Eigenschaften, welche dem direkten Einfluss der Umgebung mehr entzogen sind, verraten sich aber die Eigentümlichkeiten des Rhinocerotenstammes.

Ein ganz besonderes Interesse beanspruchen die ron Osborn als Ancylopoden zusammengefassten klauentragenden Huftiere des älteren und mittleren Tertiärs, unter denen die Chalicotherien altbekannte und weit verbreitete Formen sind. Hier kommen ganz neue, in gewissem Sinne sogar rückläufige Richtungen zum Ausdruck, allerdings nur an einem ganz beschränkten Teile des Skelettes. Vom Schädel bis zum Carpus und Tarsus herrscht der Bau des Ungulatentypus --. die Endphalangen sind scharfe, bei einigen Arten retraktile Klauen.

Nach dem gegenwärtigen Stand unserer Kenntnisse müssen diese Tiere sich schon sehr früh vom Stamme der echten Ungulaten getrennt haben, anscheinend von Formen ausgehend, die wie Menisco- 
therium noch den altertümlichen Condylarthra angehören. Eine Rückbildung der Hufe zu Klauen konnte damals noch leichter geschehen, da die Differenz noch nicht so weit wie in den jüngeren Zeiten des Tertiärs gediehen war. Immerhin bedurfte es einer ganz energischen Aenderung der ganzen Gewohnheiten, um das zu ermöglichen. Die Erfahrung lehrt, dass die rerlassene Entwickelungsrichtung die bessere war; die Ancylopoda sind sämtlich atusge-

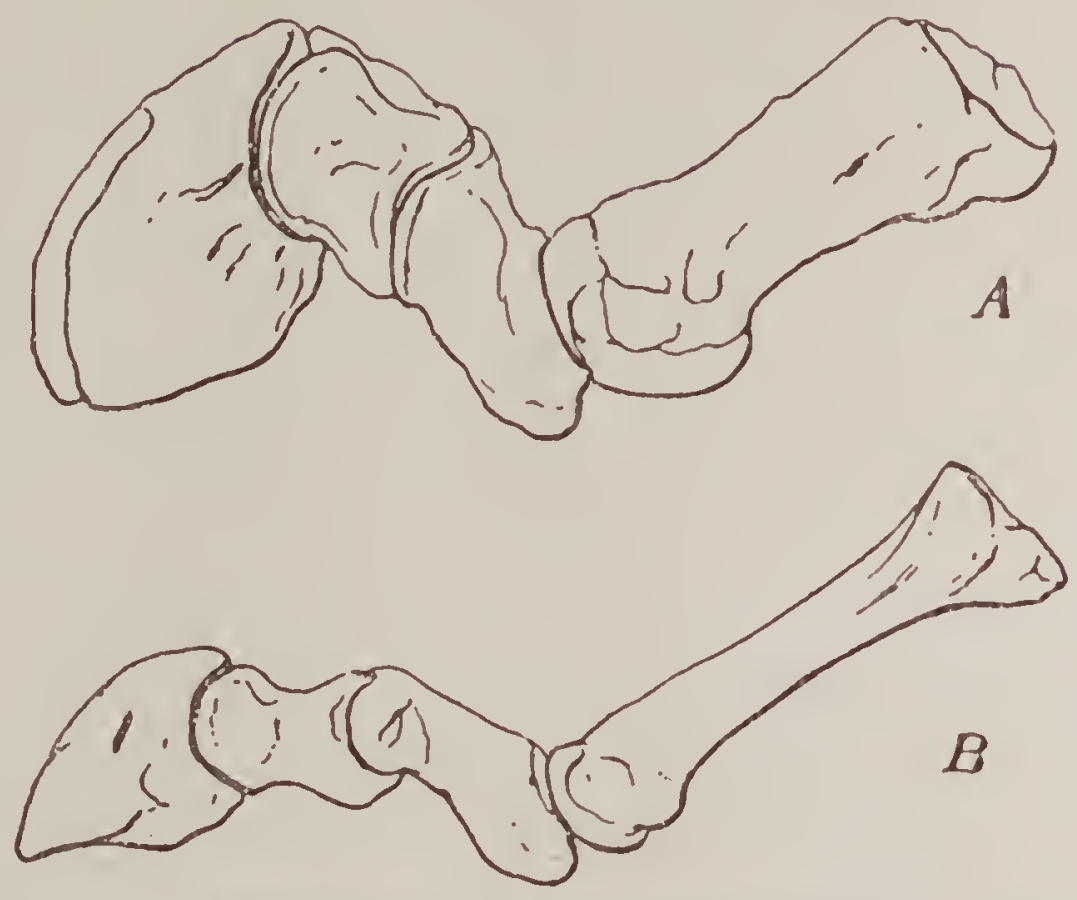

Figr. 2. Mittlerer Finger ron $A$ Chalicotherim sinnsaniense Gaudry. f) Artionyx (iaudryi ()sb. u. Wortm. storben. Nicht eine Verbesserung des Typus ist hier herausgezüchtet aus dem Ungulatenstamme, sondern Wille und fiewöhnung haben einen Erfolg herbeigefülırt, der nur den Instinkten des Tieres zu gute kam, die dauernde Existen\% der Arten aber direkt gefährdete.

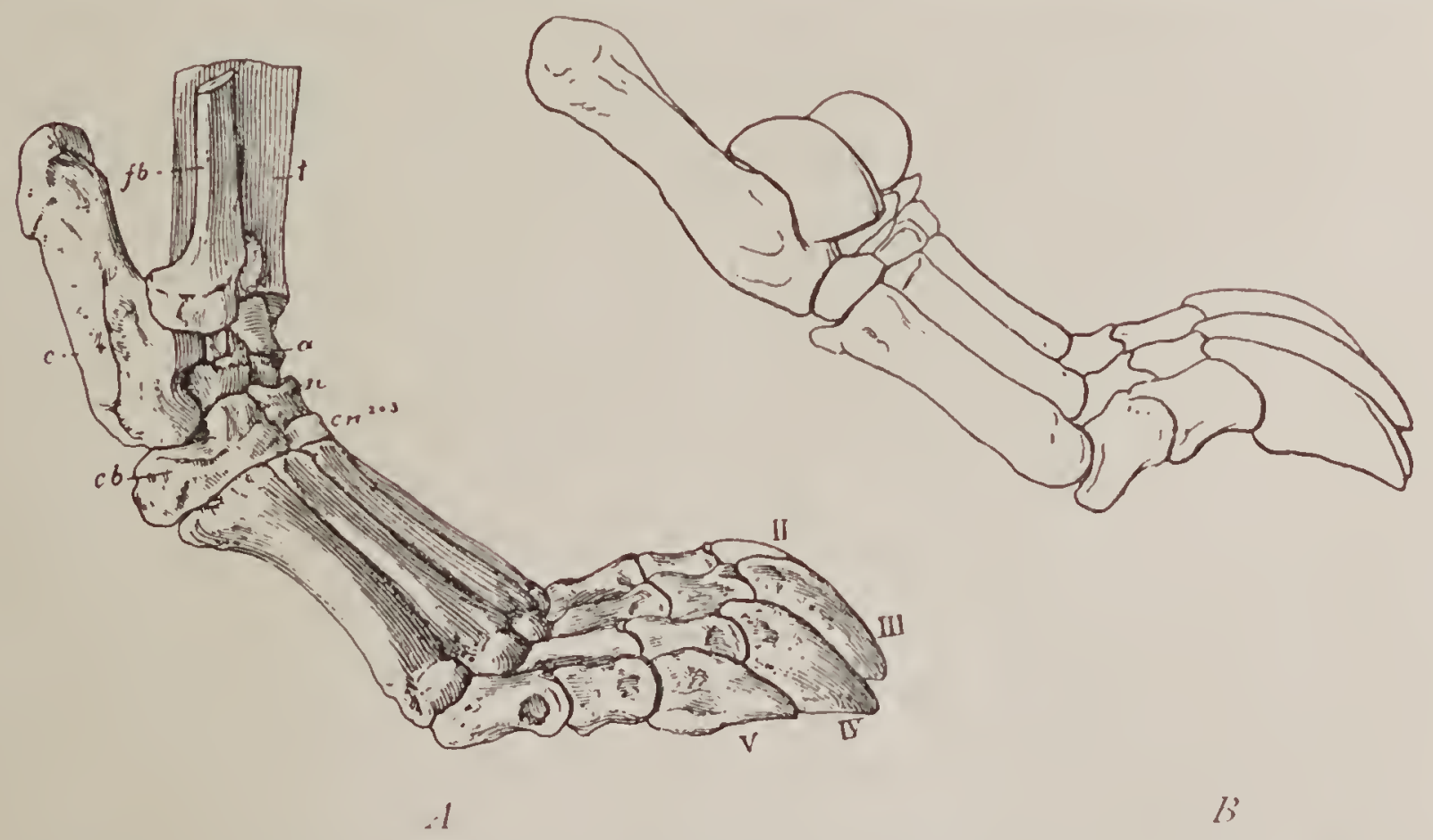

Fig. 3. Rechter Fuss, it vom Artionyx (iaudryi Osb. et Worm. If ron Chalicotherium magnum Fillıl.

Dabei ist nun noch eins bemerkenswert. Die echten Ungulata teilen sich in Perissodactyla und Artiodactyla, Paarzeher und Unpaar- 
zeher. Obwohl die Ancylopoda zu einer Zeit sich abgezweigt zu haben scheinen, wo jene Trennung noch nicht vorhanden war, bilden sich unter ihnen zwei ganz parallele Richtungen aus (Chalicotherium und Artionyx): Osborn und Wortman bezeichnen sie als Perissonychia und Artionychia. Jene, die alten Chalicotheriiden umfassend, sind Unpaarzeher, wie schon Kowalewsky vermutete; die statische Axe läuft durch Zehe III und der Tarsus ist ganz nach dem Typus der Perissodactyla gebaut; bei diesen haben wir vier Zehen und die Axe läuft zwischen Zehe III und und IV; der Tarsus ist ganz nach dem Plane der Artiodactylen gebaut. So kommen die beiden Entwickelungsrichtungen, die zu den Pferden und Ruminantiern hinleiten, auch in dieser ganz aberranten Gruppe zum Durchbruch, ja man kann sagen, dass die Eigentümlichkeiten, welche zur Aufstellung der Ordnung Ancylopoda geführt haben, einzig an den Phalangen haften, deren Anpassung das übrige Skelett völlig unberührt lässt. „If the foot of Artionyx had the metatarsals cut off halfway down, no one would hesitate to call it truly artiodactyl" (Osborn, The Ancylopoda. Americ. Naturalist 1893, S. $\left.132^{1}\right)$ ).

Wir wollen noch ein Beispiel heranholen. Nach Dollo stammen alle Marsupialier von „arboricolen“ Vorfahren; hierauf deuten der opponierbare erste Finger, die Verkleinerung des zweiten und dritten Fingers, das Ueberwiegen des vierten Fingers gegenüber jenen und häufige Syndaktylie von Finger II und III. Mit diesen ererbten Eigenschaften mussten alle Umänderungen sich ausgleichen, die später aus einer anderen Lebensweise resultierten. Neuerdings wurde der

I) Scott wies die Identität von Artionyx mit dem schon 1873 von Leidy beschriebenen Agriochoerus nach und bringt die Gattung in engere Beziehung zu den Oreodontiden, die ihrerseits mit Hyopotamiden und Anoplotheriden verwandt zu sein scheinen. Die Oreodontiden führen auf die eocänen Buno-Selenodonten zurück, und die Bridgerformation mag jene Formen ihrer Reihe enthalten, von denen dann die Agriochoeriden abzweigten; vielleicht kennt man eine solche Form schon in Helohyus. (Morpholog. Jahrb., 'XVI, 3 I 9 ff.; Proc. Amer. Philos. Soc. I894, Bd. XXXIII, p. 243 ff.)

Die Ausbildung des ganz und gar artiodactylen Baues wäre bei dieser verwandtschaftlichen Stellung weniger auffällig, da dann eine engere Beziehung zu Chalicotherium gar nicht mehr besteht. Der Parallelismus in der Ausbildung der Klauen, die im einzelnen starke Unterschiede aufweisen, ist unschwer aus der Lebensweise zu erklären, ein Parallelismus, welcher innerhalb der Ordnung Ancylopoda ausgesprochene Artiodactylie entstehen lässt, schwerer begreiflich. 
Fuss von Diprotodon australis beschrieben ${ }^{1}$ ), einer riesenhaften, fossilen Form, die sich nach Art der Pachydermen bewegte. Hier hat sich das Fussskelett in einer ganz exceptionellen Weise mit den neuen Beanspruchungen abgefunden. Gerade die Finger, welche $\%$ B. bei Huftieren die Körperlast tragen, waren während der arhoricolen Periode zur Unbrauchbarkeit geschwächt. Sie rerkümmerten noch mehr, und vom ersten Finger blieb nur der Metatarsus übrig. Ungekehrt schwollen die beanspruchten Knochen, Astragalus, Calcaneus, Tarsalia und Metatarsalia zu unförmlicher Grösse an und kompensieren den Ausfall.

Hier muss der
Wille des Tieres geradezu bestimmend eingegriffen haben, um den Verfolg einer neuen I.ebensweise, welcher die Beschaffenheit des Skeletts nicht entsprach, zu ermöglichen. Die Umgestaltungen liegen in keiner ,Richtung“, sondern sind rein adaptiv. Die Schwächung wichtiger Knochen infolge der früheren Entwickelungsrichting konnte

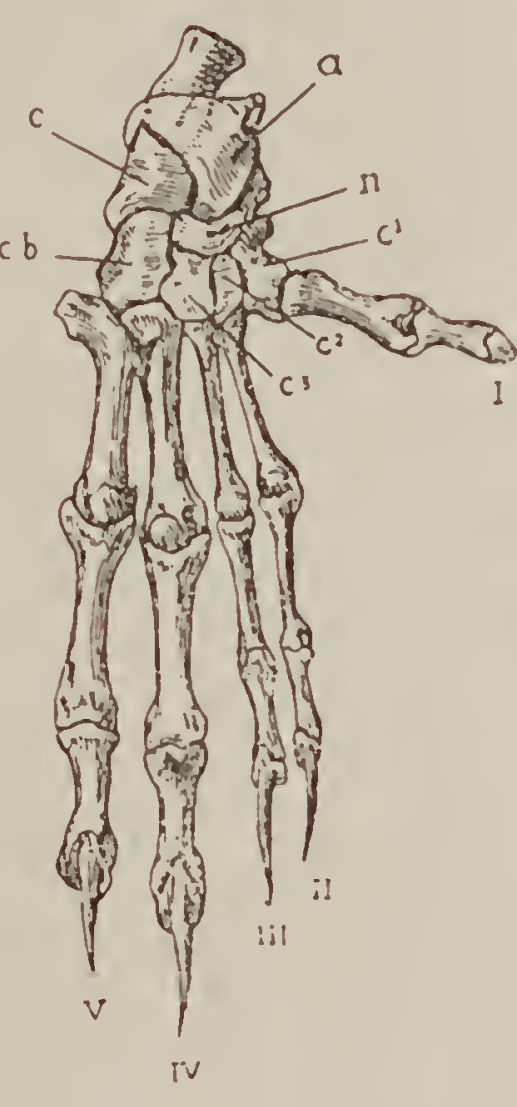

.1

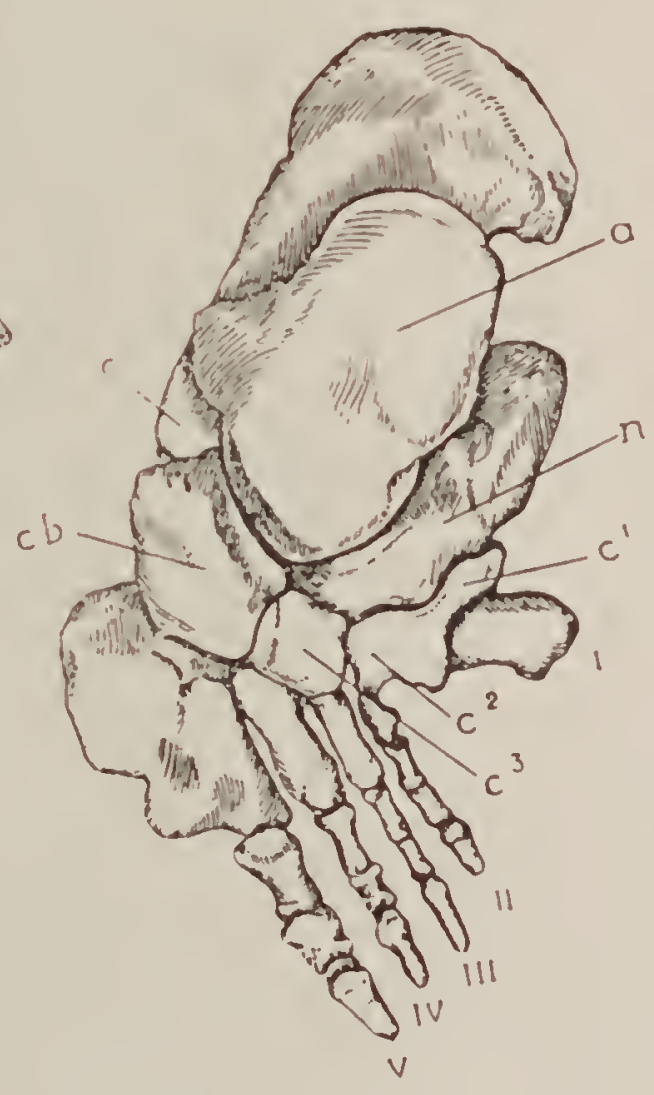

B)

Fig. 4. Rechter liuss 1 de's lebenden Trichtsurus vulpecula Kerr (Anstralien), nach lelower, $B$ des diluvialen Diprotodon australis Owen, nach Stirling n. Ziet\%. "Astragalus, i Calcaneum, cb Cuboid, $n$ Naviculare, $c^{1-3}$ dic Cunciformia. nicht wieder ausgeglichen werden und rückliegende Elemente des Fussskelettes mussten die last übernehmen. So führte die Abänderung des Fusses der Adaktylic, der Zchenlosigkeit entgegen, aber die Gattung starb aus, ehe es so weit kam.

Wir wollen nun das Thema etwas anders nehmen. Einige Linien der Huftiere sind schon im Tertiär ausgestorben und Kowa-

I) L. Dollo, Le pied du Diprotodon et l'origine arboricole des marsupiaux (Bull. scientif, de la France I 900, XXXIII, p. 278 ff.) 
lewsky führte in einer wertvollen Arbeit dies auf sog. ,inadaptive Reduktion" zurück ${ }^{1}$ ). Bei diesen Formen ist rie der Oekonomie des Körpers entsprechende Reduktion überflüssiger Zehen in anderer Weise vollzogen als bei den heute noch existierenden Linien. Die Stummel (Metacarpalia und Metatarsalia) der rudimentären Zehen beanspruchen noch einen breiten Platz am Fusse, und Carpus und Tarsus sint nicht so gut verfestigt, wie bei Hirschen und Rindern. Eigenschaften sind „gezüchtet“, welche gegenüber den Einrichtungen bei den konkurrierenden, in gleichem Sinne sich verändernden Nach-

I) Aus den Ausführungen Kowalewsky's, welche eine ganz neue Strömung in der Säugetierkunde hervorriefen und auf denen auch einige bekinnte Arbeiten ron Marsh beruhen, hebe ich nur die eine Stelle hervor, welche seine Ansichten und Beobachtungcn, wie mir scheint, am besten zusammenfasst:

„Wenn wir die Rudimente reduzierter Zehen bei den meisten fossilen Tieren, wie Anoplotherium, Xiphodon, Entelodon, Diplopus betrachten, so finden wir immer, dass dieselben eine dicke, runde, noduläre Form haben, während die Rudimente, welche bei den recenten Ungulaten vorkommen, immer sehr schmächtig und dünn sind; selbst bei Dicotyles ist das Rudiment des Metatarsale $\mathrm{V}$ immer ein langer, diinner, platter Knochen und so ist es auch bei den Hirschen und Relien. Woher kann dieser Unterschied kommen? Mir scheint er nur auf folgende Weise sich zu erklären:

Die nodulären Rudimente rühren ja sämtlich ron Formen her, die eine inadaptive Reduktion befolgen; die schmächtigen Rudimente nur von solchen, die einer adaptiven Reduktion unterworfen sind. Die inadaptive Reduktion besteht aber darin, dass alle Knochen sehr hartnäckig an ihren typischen Verhältnissen halten, dass jedes Metacarpale und Metatarsale bis zu seinem Verschwinden immer noch die ganze distale Fläche des entsprechenden Carpale und Tarsale einnimmt; wenn aber bei diesen Verhältnissen ein Seitenfinger zu schwinden beginnt, dann schwindet er ron unten leer, wo er frei ist, seine proximale Fläche aber haftet immer an dem verhältnismässig grossen Carpale und Tarsale, dessen ganze untere Facette er einnimmt; das fortgesetzte Schwinden von unten her giebt am Ende ein dickes, noduläres Rudiment, das immer noch an der ganzen distalen Fläche seines Carpale oder Tarsale haftet, wie wir es beim Anoplotherium, Xiphodon, Entelodon sehen. Im Gegenteil, hei den Ruminanten und Suiden, die eine adaptive Reduktion befolgen, wird bei der Vergrösserung der Mittelzehen nicht auf die typischen Verhältnisse geachtet, Traditionen scheinen da keinen Einfluss zu besitzen, das sicli ausbreitende III. oder IV. Metacarpale (oder Metatarsale) greift auf die typische Fläche eines Seitenfingers über, eignet sich einen Teil dieser Fläche an; dadurch aber wird die proximale Fläche des Seitenfingers verengt und infolgedessen muss der Seitenfinger in seiner ganzen Länge sich verdünnen; der Prozess geht immer in derselben Richtung weiter die proximale Fläche des Seitenfingers an seinem typischen Carpale oder Tarsale wird inmer enger, Millimeter um Millimeter rückt der 3. Finger nach und um so viel verengt sich der Seitenfinger; die Verengung an der proximalen Fïäche setzt sich als Verdünnung auf die ganze Länge des Seitenfingers fort; endlich bleibt ihm oben nichts mehr übrig als ein Punlit (wie bei den Traguliden) und die Seitenzehe wird fadenförmig“ (1873, Monographic der Gattung Anthracotherium. Palacontographica XXII, S. 196). 
barlinien inferior genannt werden müssen. Die ,inadaptiv reduzierten" Huftiere sind allerdings ausgestorben, aber sie haben doch längere Zeit hindurch sich entwickelt und jene Eigenschaften accentuiert. Nach einem Hauptsat\% der Darwin'schen Lehre konnte bei herrschender Konkurrenz eine Weiterentwickelung ,inadaptiver Eigenschaften" gar nicht stattfinden.

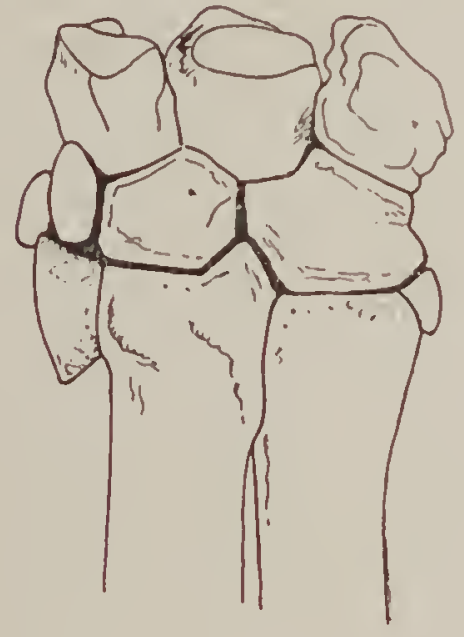

$A$

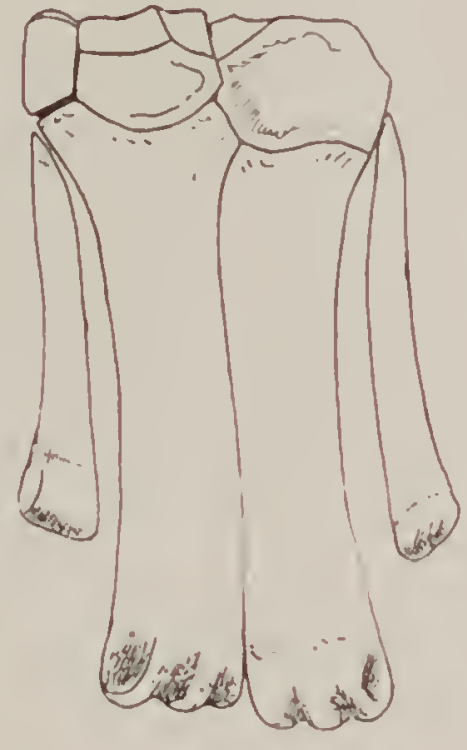

b)

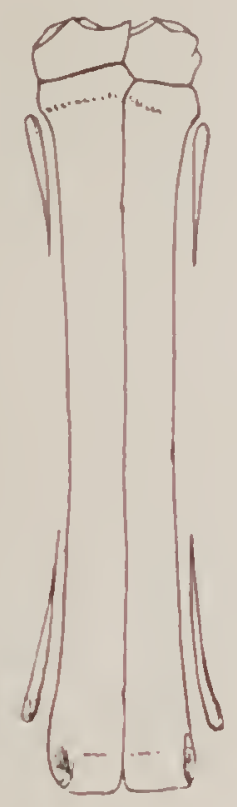

$C^{*}$

Fig. 5. A Fussskelett ron Anoplotherium (Unteroligocin), inadaptiv reduziert; If Fusmskelett von Dicotyles (recent), $C$ von Gelocus (Oligociin), mit unvollstïndiger aber adaptiver Redultion der Seitenzehen (nach Kowalersliy).

Wir verstehen die Fälle, in denen Eigenschaften weiter entwickelt werden, welche geringerer Qualität sind als die homologen Merkmale gleichzeitiger Konkurrenten, vielleicht eher, wenn wir uns vorher in die Erinnerung zurückrufen, wie oft Eigenschaften zunehmen und abschwellen, bei denen eine Verknüpfung mit dem Nutzen gar nicht ins spiel kommt.

Die wunderbare Formenfülle der Radiolarienskelette, die Mannigfaltigkeit der Schwammskelette, insbesondere der Obertlächennadeln, die Gattungen der palaeozoischen Tabulaten-Korallen verdienten wohl in diesem sinne eine ausführliche Besprechung ${ }^{1}$ ).

I) Ich möchte hier auf eine treffende Bemerliung hinweisen, welche Dall in seiner Beschreibung der Blake Mollusca macht (Blake mollusca, p. I 26): "The absence of struggle which characterizes life in deeps as opposed to that of the shores, and which is illustrated by the absence of the protective operculum in so many species (nämlich Gastropoden), does not limit the variations of external form as they are limited by economical and other reasons where the struggle is intense. In estimating the archibenthal faune this must steadily be kept in view." 
Nach Darwin's Prinzip können physiologisch indifferente oder nutzlose Merkmale nicht erhalten bleiben, nach dem Sparsamkeitsprinzip müssten sie verkümmern. Was bedeutet aber der auffallend grosse Sacculus-Otolith für das Gehörorgan des in Holothurien eng eingeschlossen schmarotzenden Bandfisches, welche Funktion wiederum soll er noch ausüben bei den in lautlosen abyssischen Tiefen lebenden Macruriden?

Eine fast kontinuierliche Reihe führt von den nucleaten Terebrateln hinüber zu der auffallenden Pygope mit Durchbohrung beider Schalen; eine ganz analoge Abstufung verbindet Clypeaster mit Scutella und Amphiope. Eigenschaften steigern sich hier, als würden sie in einer Richtung gezüchtet; aber soll man glauben, dass der Kampf ums Dasein, die natürliche Züchtung die Leitung übernommen hat? Hier versagen auch die auxiliaren Prinzipien und die I amarcksche Anpassungslehre. Die Metamorphose der Schalen, des Skelettes vollzieht sich in einer Unabhängigkeit, die in der 'That an ,.organisches Wachsen" infolge irgend eines Reizes mahnt, und sie beharrt in einer Richtung, bis sie mit einem anderen Prinzip interferiert oder in Kollision kommt. Hier kann man auch an die Geweihbildungen der Hirsche erinnern, welche bei Polycladus und Euryceros als entschieden hypertroph bezeichnet werden müssen und schwerlich selektiv nach dem Nutzen herausgezüchtet sind.

Ich verweise ferner auf die den Körper überlastende Enntwickelung des Hautpanzers bei Stegosauriern. Die auf dem Schwanze inserierenden Stachelplatten mögen als Waffen einen Nutzen gehabt haben, obwohl gleichzeitige Dinosaurier mit besseren Waffen ausgerüstet sind, aber die Riesenplatten über der Mittellinie des Körpers sind Wucherungen und Ballast für den so sparsamen tierischen Körper.

Diese Reihen der gewaltig grossen Dinosaurier, deren Bewegung auf dem festen Lande enormen Kraftaufwand bedingt und viel mehr Kraft verbraucht, als die Bewegung der zum Teil ja noch grösseren Cetaceen im Wasser, diese Dinosaurier, deren Gehirn dazu so klein war, dass es durch den Rückenmarkskanal gezogen werden konnte, treiben dem Untergange entgegen. Der 
Kampf ums Dasein, oder richtiger die allgemeine Konkurrenz, mag ihre Tage verkürzt haben, aber gezählt waren sie ohnedies, sobald

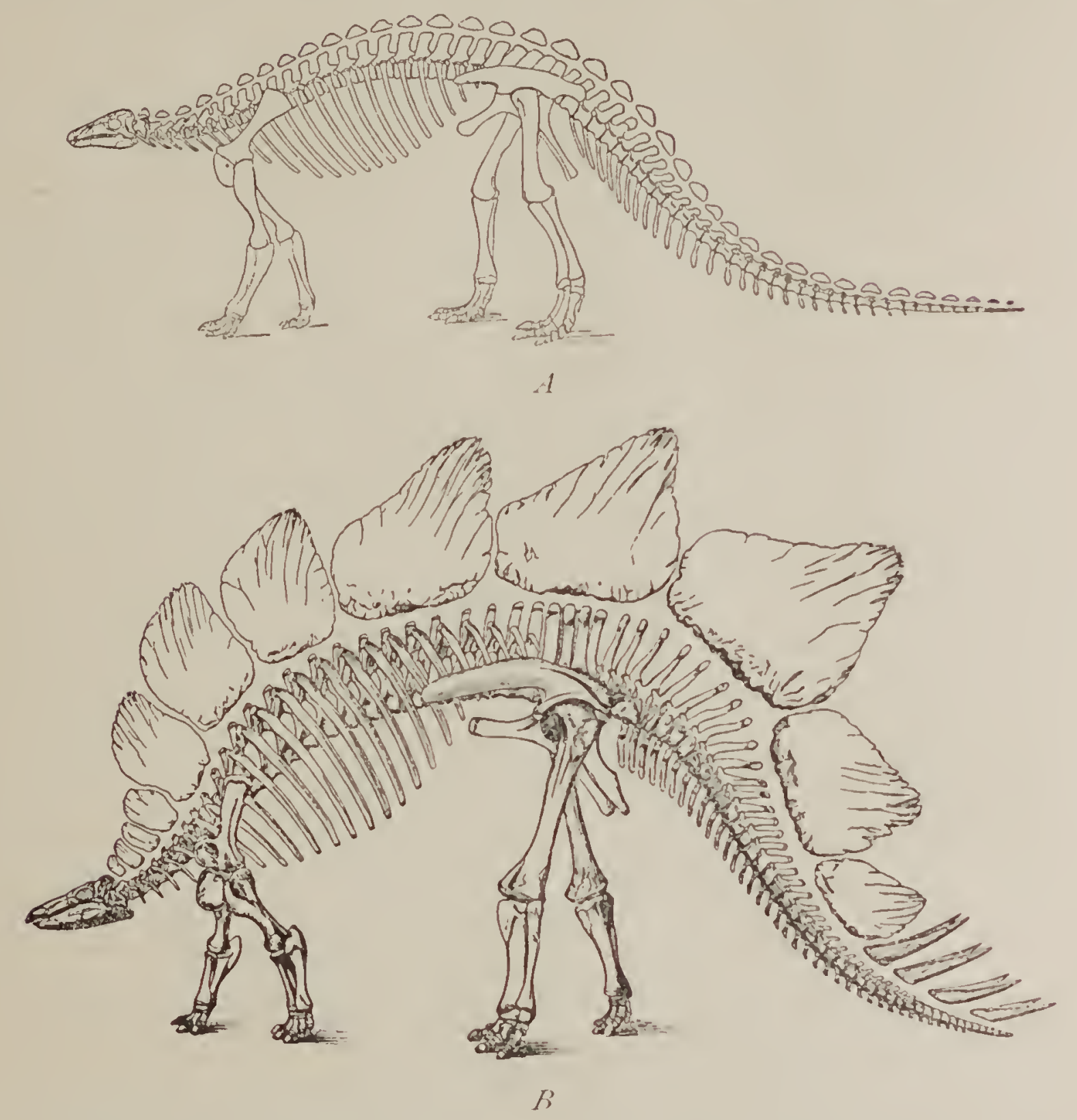

Fig. 6. A Scelidosaurus Harrisoni Owen; Lias, England. 1/36 11. Gr. $B$ Stegosaurus ungulatus Marsh. Oberster Jura (Atlantosaurus-beds). Colorado. '/60 11. Gr.

die gefährliche Richtung ihrer Entwickelung eine umgehemmte wurde. Kein Kampf ums Dasein hat diese Entwickelung gehemmt. die mit Scelidosaurus im Lias beginnt und sich bis in die Kreide fortzieht.

Wir wollen hier Halt machen, obwohl noch vieles hervorzuheben wäre, insbesondere die Bedeutung des biogenetischen Gesetzes in der Palaeontologie ${ }^{1}$ ) und die bei niederen Tieren vorliegrende Möglichkeit,

I) Die amerikanischen Palaeontologen legen ein grosses Gewicht auf die sog. ontogenetische Methode, d. h. man überträgt die von Biologen ausgearbeitete Art zu schliessen auf das fossile Material, soweit uns neben der erwachsenen Form auch ihre Jugendzustände aufbewahrt sind. So kennt man fast die ganze Entwickelung einiger Trilobiten, vieler Cephalopoden, Brachiopoden etc. Aus je älteren Schichten die Art entnommen ist, deren Jugendstadien 
auf einem sonst vorübergehenden Zustand der Ontogenie stehen zu bleiben und in ihm einen Ausgangspunkt für eine neue Entwickelungs-

wir zergliedern, desto mehr können wir hoffen, die Ontogenie von den störenden Einwirkungen der Tachygenesis, der Ablürzung, der Fälschung etc. befreit zu finden.

In allen Fällen stossen wir aber auf einen unlöslichen Rückstand, d. b. gerade dort, wo die Methode uns über die Resultate, welche die bisherige Palaeontologie lieferte, erheben sollte, hört sie auf, anwendbar zu sein. Mit dem Protoconch der Ammoniten lässt sich nichts weiter anfangen. Das eigentlich embryonale Leben, das sich 1 dieser Hülle abspielte, bleibt uns ewig unbekannt, und die Hülle selbst zeigt in ihren an sich so einfachen Charakteren doch so viele Schwankungen, dass wir nicht einmal eine Ruhe im Einfachsten sehen, sondern finden, wie auch dies den abändernden Einflüssen von je her unterlegen ist. Auffälliger noch sind die Schwankungen, welche das Embryonalgehäuse der Gastropoden zeigt.

Wenn die erste Kammerscheidewand eine einfache ist, die des ausgewachsenen Tieres in zerschlitzte Loben und Sättel zerlegt ist, so müssen diese weit anseinanderliegenden Zustände in der Ontogenie durch Zwischenstadien vermittelt werden, wie wir beim Zerlegen eines jeden Ammoniten selıen. Für eine speziellere Phylogenie beweisen die Uebergangssuturen zunächst nichts, deun auch wenn keine Descendenz die Arten verbände oder keine Rekapitulation der Phylogenie vorläge, müssten sie sich in einer kontinuierlichen Reihe folgen. Wir würden kein Recht haben, auf die Abstammung eines Aegoceras von gephyrocerasartigen Goniatiten zu schliessen, wenn uns nicht entsprechende Typen aus den in Frage kommenden Erdperioden vorlägen. Der phylogenetische Wert der ontogenetischen Entwickelungsstadien bedarf anch hier im Gebiete der Palaeontologie selbst wieder der Bestätigung durch andere Resultate der Palaeontologie, d. h. wir kommeu keinen Schritt weiter. Und dabei ist der Schritt ein so minimaler von Goniatiten zum Ammoniten, dreht sich das Ganze um eine Spanne des allgemeinen Entwickelungsganges, den die tetrabranchiaten Cephalopoden durchgemacht haben.

Auffallend ist nur, dass man nicht häufiger einen anderen Schluss gezogen hat, der mir notwendig scheint, dass nämlich die Achulichlieit der ontogenetischen Entwickelung, welche eine gerichtete und eingeengte ist, mit der geologischen Aufeinanderfolge der 'Typen (z. B. Goniatites - Ammonites) auch für die phylogenetische Entwickelung, wenn nicht das Vorhandensein einer bestimmteu 'Tendenz erfordert, so doch das Feld des Zufälligen einengt.

Greifen wir noch auf die ontogennetische Entwickelung einer anderen Gruppe zurück, nämlich die der Trilobiten, welche durch Barrande seit langer Zeit für einige Arten bekannt ist. Durch amerikanische Forscher ist sic auch für andere Arten nachgewiesen, so dass wenigstens aus den Hauptgruppen der formenreichen Abteilung Material rorliegt. Jede Ontogenie beginnt, nach überwındenen Eistadium (dem wir auch hier nicht näher beikommen können) mit recht einfachen Formen, welche Beecher das Protaspis-Stadium neunt; aber auch diese einfachen Formen, über die wir unsere Untersuchungen nicht hinausdehnen können, bieten Verschiedenheiten. Protaspis ist für die amerilianischen Forscher der „Phylembryo" der Trilobiten und zugleich ein larvaler Zustand, welcher dem hypothetischen Protonauplius der prinitiven, ancestralen, larvalen Form aller Crustaceen sehr wahe stelit. Es ist nun gewiss folgerichtig, wenn Beecher den aus dem Cambrium bekannten Protaspis-Zuständen diejenigen als die primitivsten, am wenigsten ,,ïberarbeiteten“ heraussucht, welche die einfachsten Merkmale zeigen und zwar nur solche, welche auch bei allen anderen Protaspisformen wiederkelıren. Und doch nuss hier ein Trugschluss vorliegen, 


\section{richtung zu finden ${ }^{1}$. Auch dass die Artbildung in den verschiedenen}

denn der bewährte Tribobitenforscher liommt dadurch zu der völlig unhaltbaren Ansicht, dass dic Trilobiten ron blinden Vorfahren abstammen.

Eine Beobachtung will ich dagegen anführen, es ist die, dass der in Alter blinde Trinucleus im frühesten Jugendstadium Augen zeigt. Wenn wir die Ontogenie verwerten wollen, so ist hier ein Punkt, richtig cinzusetzen. Wir erfahren dadurch, was uns die breite Entfaltung der Klasse lehrt, dass bei blinden Formen die Augen rückgebildet sind. Das abstrahierende Verfahren, immer das Einfachste anfousuchen als Ausgangspunlat der Entwickelnngen, würcle irre leiten.

So fürclite ich, dass wir anch mit Benutzung der Ontogenie der ältesten belsannten Formen, selhst Wenn das Naterial sich enorm rerrollständligt. nicht in das Dunkel sehen lä̈nnen, welches üluer den präcambrischen Generationen lagert. Bis an diese Grenze leitet uns aber die zcitliche Aufeinanderfolge realer Wresen, die Jieisch, Blut und Skeiett gewesen sind, sicherer, als dis hiogenetische (ieset/. Der Went, der in dem zeitlieh greordneton Lrkundenmaterial steckt, muss hïher angeschlagen werden.

Zweifellos gicht es in Verlanfe der Ontegrenese \%ustinde, welche I.icht auf den Entwickelungsgnng des Stammes werfen. So wenn sich vorüluergehend ein ()rgan höher ausgebildet zeigt, als e's spät r hein biwachsenen auftritt, wenn Orgatne vorhanelen sind, die später alsorbient werden, wemn cin Organ durch cinen liomplizierten Zustand in cinen ('infachen hineingleitet - denn derartige Zustinde kann die von Einfachen konsecpuent fortschreitende Entwickelnngstendenz allein nicht crklären, das sind Zuhaten der spentellen Stammesgeschichte. Diesen Beobachtungen kommt der grosse Went \%u, dass sic uns das Recht geben, analog heschaffene fossile formen, auch weun sie sporadisch aufueten und durch weite Lüelien getrennt sind, in Entwiclielungsreihen, welche zu den lebenden hinüberführen, einzustellen. Es sei erinnert an Archneopteryx und die Zalnüögel der Kreide, deren sicherer Platz im System wesentlich auf der richtigen Ausmutzung embryologischer Erfahrung beruht. Ueber eine gewisse Entfernung tragen aher diese Erinnermugen in der Ontogenie nicht hinaus, Wohl nie üher die Grenzen einer KKlasse des \%oologischen Systems.

Man betont dagegen das Auftreten der Kiemenspalten des Fisches noch in der On. togenie des Säugetieres. Aber es ist schon mehrfacl ausgesprochen, dass die Fische nicht notwendig primitive Formen sein müssen, sondern auch ron küstenbewohnenden Landtieren abstammen können. Ich will nicht behaupten, dass es erwiesen sei, aber mindestens ist diese Annahme ebenso möglich wie die entgegengesetzte. Die Kluft zwischen ihnen und den Stegocephalen ist durch keinen Fischtypus, auch nicht durch den der Coelacanthinen zu ïberbrücken. Die Struktur der Flosse lleibt fundawental verschicden von jener der Eixtremitäten höherer Vertcbraten; der Unterschied würde verständlicher, wenn wir annehmen, dass sie in sebr alten Zeiten von Tetrapoden mit Knorpelskelett abzweigten. Die Anforderungen an die Bewegung, welche das Lehen iul Wasser stellt, arbeiteten die noch einheitlich knorpeligen, durch keine oder wenige Ossifizierungen ausgezeichneten Kunorpelstiicke des Skeletts in radikaler Weise um.

Diejenigen Stegocephalen des Perms, welche ein kiementragendes Stadium durchmachen, sind nur ein kleiner Teil dieser bedeutenden Gruppe; ihre Kiemen sind eine neue Erwerbung, physiologisch und morphologisch etwas anderes als die Fischliemen.

Der angedeutete Entwicklungsgang der Fische wïrdc also nach ciner ganz anderen Richtung vom Ausgangspunkte fülıren, als die der Stegocephalen, Reptilien und Säugetiere. Wenn einmal triftigere Gründe hierfür vorliegen, kann das Auftreten sog. Kiementaschen im Embryo keinen Gegengrund bilden.

I) Besonders Jaeckel hat in seiner umfassenden Darstellung der Cystoidea für die 
Phylen mit verschiedenen Mitteln arbeitet, dass durch die höhere Ausbildung der Instinkte und des Wollens bei höheren Typen sich ihr mehr Handhaben bieten, verdiente eine vollere Besprechung.

Das Darwin'sche Prinzip der Selektion ist nicht das einzige, das in Betracht kommt und es scheint nicht das wichtigste zu sein. Vielfach vermissen wir in der palaeontologischen Geschichte den Hinweis auf ein Eingreifen des Kampfes ums Dasein und andererseits heben sich Richtungen der Entwickelung heraus, welche nicht in Beziehung zu einem Nutzen stehen, in einigen Fällen zu einer Schädigung der socialen Bedingungen führen.

Aus dem biogenetischen Gesetz liest man heraus, dass die Ontogenie im allgemeinen die Stammesgeschichte rekapituliert. Die Stammesgeschichte aber lehrt uns, dass auch sie nicht planlos verläuft, sondern durch das Ausgangsmaterial gerichtet ist, ähnlich wie die Ontogenie durch das Plasma der Eizelle. A ehnlich nur, denn es ist nicht ein festes Ziel, dem ein Organismus durch die Entwickelung entgegentreibt, sondern ein reicher Komplex von Möglichkeiten. Das ist für uns die Bedeutung der Konstitution. Verschieden wirken die Umgebungsreize, Anpassung, Ernährung, Selektion auf die Arten ein; sie können die Richtung der Entwickelung ändern und hemmen, sie können die Entwickelungsbahnen von Arten, die getrennten Stammes sind, einander bis fast zur Berührung nähern, eine Verschmelzung wird durch die Konstitution gehindert.

Dass es Perioden giebt, in denen die Artbildung rascher arbeitet, scheint aus der Gruppierung des palaeontologischen Materials hervorzugehen, obwohl gewisse Fehlerquellen (Iücken-des geologischen Berichtes, Transgression der Faunen) dem abwägenden Urteil nicht entgehen können. Die hervorgehobenen Erscheinungen, dass grosse

Möglichkeit eines solchen Entwickehnngsøanges Material beigebracht. Ich citiere nur eine Stelle, welche für seine Auffassung charakteristisch ist: „Die für die ganze Klärung entscheidende Supposition, dass die Sphaeroniden durch Entwickelungshemmung entstanden seien, giebt auch zugleich die Erlklärung dafür, dass ihre jüngeren Nachkommen im Gegensatz zu dem Gros der Dichoporiten z. T. aufsteigende Entwickelungsreihen bildeten. Die Indifferenz ontogenetisch früher Stadien verschaffte ihnen unter obiger Annahme leicht die Möglichkeit, beim Ausbau ihres neuen Hauses Fehler zu meiden, von denen sich die Ontogenie der Dichoporiten anscheinend nicht mehr zu befreien vermochte." (Stammesgeschichte der Pelmatozoen, I, S. 37 I.) 
Gruppen sich ohne längere Vorbereitung einstellen, dass kontinuierliche Reihen relativ kurz sind, dass manche Gattungen und Familien eine kurze auffallende Blütezeit erleben (Fusulina, Nummulites), reihen sich alle unter diesem Gesichtspunkte ein und es kann nur eine äussere Ursache sein, welche dies veranlasste.

Wann es uns gelingen wird -. und ob jemals - einen tieferen Einblick zu gewinnen, ob jemals diese so weit zurückliegenden Torgänge sich uns entschleiern, ist mehr als unsicher. Es ist aber auch ganz ungewiss, ob es gerade die imposanten Ereignisse, wie die Gebirgsbildungen und die periodisch gesteigerten Ausserungen rulkanischer Thätigkeit sind, die hier in Betracht kommen, oder mehr die Umänderungen der kontinentalen Umrisse und des Klimas, aber etwas Reales scheint zu Grunde zu liegen.

Seit den ältesten cambrischen Zeiten, ron denen her unsere Urkunden datieren, sind auf der Erde dieselben geolngischen Faktoren thätig. An den Salzlagern Indiens können wir die chemische \%usammensetzung des Meereswassers der cambrischen Zeit prüfen und wir erfahren, dass es dieselben Stoffe sind. Welche noch heute den Rückstand des oceanischen Wassers bilden. Die für das Leben nötige Wärme kam von je her nicht aus der Tiefe der Erde, sondern ist ein Geschenk der Sonne, die IVärme und Licht in gleicher Menge beständig über die Länder zerstreute. Aber die Umrisse der Festländer und Mrere haben sich geändert und ihren Aenderungen folgten solche des Klimas. Die Pole der Erde hüllten sich seit dem Ende des Tertiärs in Eis, während sie rorher oftmals das Gebiet üppigen Pflanzenwuchses waren. Daraus den Schluss zu ziehen, dass einer warmen polaren Gegend eine ausserordentlich heisse subtropische und tropische Zone entsprochen habe und demnach, dass damals die Gesamtzufuhr an Wärme durch die Sonne eine grössere war, wäre nicht richtig. Die Verteilung von Wasser und Erde als absorbierende und strahlende Flächen gegenüber dem Sonnenlicht ist von ausschlaggebender Bedeutung: Nach dem Pflanzenwuchs der Steinkohlenzeit zu schliessen, umspannten damals gleichförmige klimatische Bedingungen die ganze nördliche Hemisphäre; wo immer Steinkohlenflötze vorhanden sind, werden sie ron denselben oder nahe rerwandten Pflanzenarten be- 
gleitet. Dieses gleichförmige Klima konnte aber nicht das der Tropen sein, denn ein solches verhindert jede Aufspeicherung vegetabilischer Brennstoffe. Unzweideutige Spuren des Frostes und des Eises, ja einer grossen Eiszeit durchsetzen die permischen kohlenführenden Schichten der südlichen Hemisphäre.

Hebungen und Senkungen der Kontinente, Einbrüche, Auftreibungen und Faltungen innerhalb dieser breiten Flächen, Umwandlung einer Inselwelt in ein grosses Festland, Zersplitterung eines Kontinents in einzelne, von seichten Meeren umflossene Inseln, Ansammlung ausgedehnter Binnenmeere in Depressionen der Kontinente - alles dieses in beständiger, sich kreuzender Bewegung - bilden das Leben der Erde. Und dieses muss bedeutungsvoll nicht nur für die Verteilung der Organismen, sondern auch für die Artbildung und Descendenz sein. An Versuchen, solchen geologischen Vorgängen nachzuspüren, welche in den Entwickelungsgang der Organismen stärker eingegriffen haben und bestimmte Beispiele für solche Wechselbeziehungen herauszufinden, hat es nicht gefehlt, doch kann man nicht sagen, dass sie von besonderem Erfolg begleitet gewesen sind. Zum Teil waren die Unterlagen zu schwach, zum Teil handelt es sich auch nur um ebenso kühne als naive Gedanken, die mehr auf das Wohlwollen eines phantasieliebenden Publikums als auf eine thatsächliche Förderung unserer Erkenntnis berechnet waren.

Die Flora, welche um die Wende der palaeozoischen Zeit auftritt und nach der Häufigkeit der Gattung Glossopteris als Glossopterisflora bezeichnet wird, mag als Mutterflora der späteren triassischen und jurassischen aufgefasst werden können, aber ganz zweifelhaft ist ihre Beziehung zu der „carbonen“ Eiszeit, deren Spuren den permischen Schichten der südlichen Hemisphäre so häufig eingeprägt sind. Waagen sah in ihr die auf alpinen Höhen jener Zeit entstandene Flora, allein seither kennt man sie in weltweiter, zeitlich und örtlich von den carbonisch-permischen Hochgebirgen unabhängiger Verbreitung von Südafrika bis zu den eisigen Ufern der nördlichen Dwina.

Sind diese Konjekturen einer wissenschaftlichen Erörterung fähig und würdig, ernsthaft $z u$ nehmende Ansätze zu einer erklärenden Palaeontologie, so sind die Versuche, die Entstehung der behaarten 
Säugetiere und den Uebergang von der Oviparität zu der placcntalen Gravidität mit dem vermuteten klimatischen Umschwung jener Zeit in Verbindung zu bringen, vorläufig freie Phantasien.

Selbst die in ihren Folgen für die Ausgestaltung unserer Länder so wichtige diluviale Eiszeit hat die Entwickelung der Organismen in keine neuen Bahnen gelenkt; wir beobachten nur eine tiefgreifende Beunruhigung der Organismen, ein rastloses Wandern, Fliehen und Zurückfluten, wobei viele Existenzen vernichtet, viele Ansiedelungen zerstört und neue Gruppierungen angeregt wurden. Von grosser Tragweite ist die Eiszeit allerdings für die Entwickelung der menschlichen Kultur geworden.

Eine wichtigere Beziehung besteht zwischen dem Auftreten und Aufblühen des Stammes der Huftiere und den Veränderungen, welche ihre nordamerikanische Heimat während des älteren Tertiärs betrafen. Die Einengung der grossen Seen, die Ausbildung weiter, mit harten Gräsern bestandener Weideflächen schuf Bedingungen, denen sowohl das Extremitätenskelett wie auch die Bezahnung jener Tiere mechanisch angepasst erscheinen. Es wäre verfrüht, hier eine Abhängigkeit vorauszusetzen, aber auch das Nebeneinander der Vorgänge ist bedeutungsvoll genug und kann uns ermutigen, dem Zusammenhange weiter nachzuspüren. Die Bedeutung des geologischen Vorganges liegt darin, dass neue Lebensgebiete, neue Möglichkeiten, zu leben, erschlossen wurden, während die Auftürmung alpiner Gebirge oder die Vereisung weiter Fä̀chen beides einschränkt. Jenes regt die Instinkte, den Willen der Tiere an und zieht die Gewöhnung und damit die Anpassung nach sich, dieses führt zunächst zu einem brutalen Kampfe um die Existenz, der für die morphologische Ausbildung viel weniger befruchtend wirkt. 


\section{Druckfehlerberichtigung.}

S. 5 Z. $\mathrm{x} 2$ v. o. vergleichen statt verghenleic.

S. 6 2. x I v. o. dic statt der.

S. 12 Z. 7 v. o. Valaeospondylus statt Urocordylus.

S. 23 Fig. 4, Unterschrift Trichusurus vuipecula statr Trichosurus vulpecul.

S. 28 Z. 4 v. u. unter statt mit.

S. 33 Z. 5 v. n. Flächen statt Elächen. 